\title{
HAEMORRHAGE INTO THE LENS* A COMPLICATION OF INTRA-OCULAR SURGERY
}

\author{
BY \\ P. R. STEVENS AND S. CHATTERJEE \\ From the University Department of Ophthalmology, and Royal Eye Hospital, Manchester
}

HAEMORRHAGE into the crystalline lens as the solitary complication of an intra-ocular operation must be an exceptional event, but we have recently observed two such cases. Both patients were treated surgically for glaucoma and blood was seen in the lens shortly after operation.

\section{Case Reports}

Case 1, a man aged 55 years, attended hospital on November 10, 1956, complaining of pain in the left eye; 5 weeks previously he had been treated with atropine for a corneal ulcer but this had been discontinued 2 weeks before the onset of the pain.

Examination.-He had a shallow anterior chamber, a semi-dilated sluggish pupil, and a pathologically cupped optic disc. The intra-ocular pressure was $45 \mathrm{~mm}$. Hg Schiötz, and gonioscopy showed a narrow angle, open above but closed below. The corrected visual acuity was $6 / 18$ in the left eye and 6/6 in the right, which was in all respects healthy.

Treatment.-Gutt. pilocarpine 1 per cent. four times a day failed to control the intraocular pressure, which varied between 45 and $79 \mathrm{~mm}$. Hg Schiötz. On December 10, 1956, a filtration operation was performed, consisting of combined anterior flap sclerotomy, cyclodialysis, and basal iris inclusion (Stallard, 1953). There were apparently no operative complications.

On the sixth post-operative day the fundus reflex was lost and a haemorrhage was noted "on the surface" of the lens, but slit-lamp examination later the same day revealed the haemorrhage to be within the lens. There was no trace of hyphaema in the anterior chamber. The anterior surface of the lens appeared intact, there being no visible tear in the lens capsule. The haemorrhage had spread down from the upper nasal quadrant covering the whole of the pupillary area except for a narrow crescentic space on the infero-temporal side through which a faint fundus reflex could be obtained. The edges of further blood films were noted in the deeper layers. The lens sutures were seen as dark lines against the lower edges of these sheets of haemorrhage. Deeper parts of the anterior cortex and nucleus were obscured from view except in the infero-temporal quadrant where they were free from blood (Fig. 1 opposite).

* Received for publication December 16, 1957. 

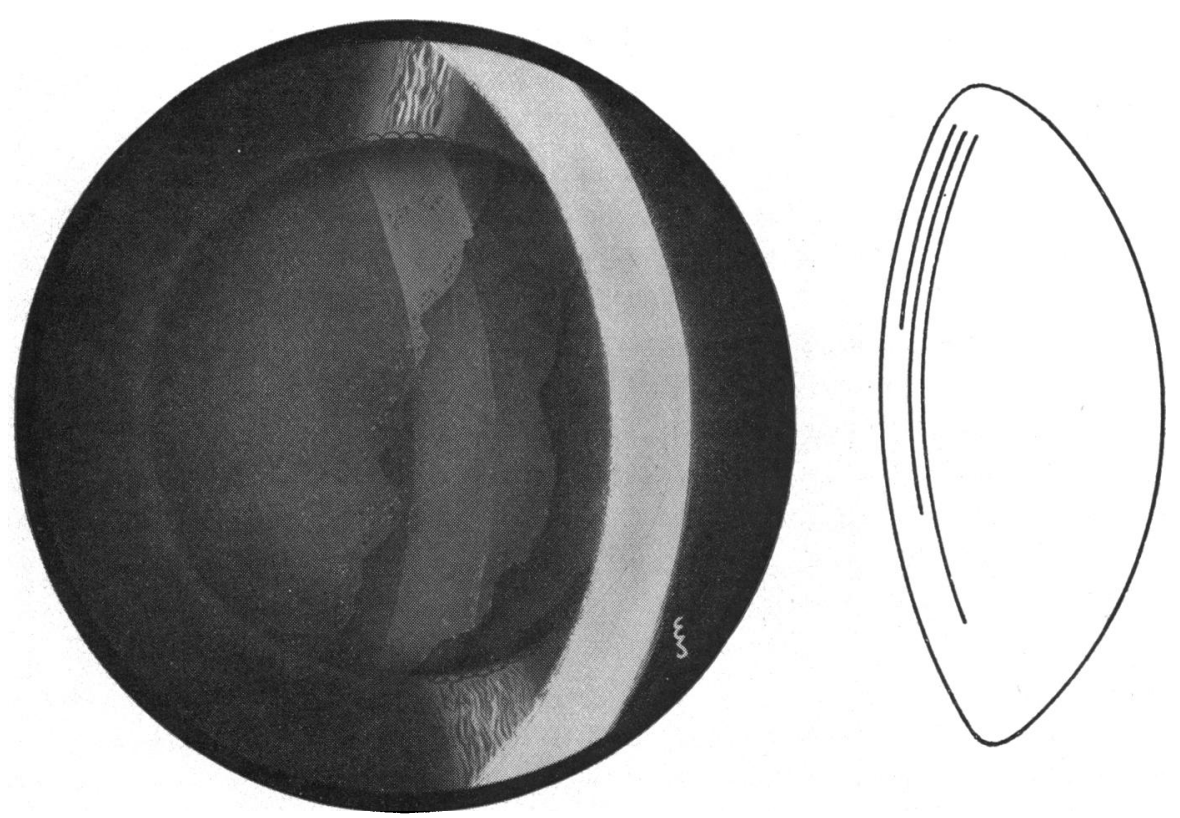

Fig. 1.-Layers of haemorrhage in the anterior cortex of the left lens of Case 1.

Follow-up.-Gonioscopic examination 3 months later showed that the iridotomy was closed by fibrous tissue, and after 11 months there was very little change in the condition except for the appearance of a few dot opacities in the clear portion of the cortex and a suggestion of clearing of the anterior film of blood on the temporal side.

Case 2, a woman aged 67 years, was admitted to hospital on April 6, 1957, with a subacute congestive attack of glaucoma in the right eye which was rapidly controlled by eserine and Diamox.

Past History.-In November, 1953, the patient had an iridectomy following a severe attack of acute congestive glaucoma in the left eye, and subsequently an iris inclusion was performed as the iridectomy had failed to control the ocular tension adequately. She had been under observation in the Glaucoma Clinic since her first attendance, and the unoperated right eye had remained symptomless and had shown no positive signs of progressive glaucoma although the optic disc was reported as "cupped". The right visual field remained full and there was no central field defect; the ocular tension was invariably $28 \mathrm{~mm}$. Hg Schiötz (recorded in the early afternoon); corrected vision remained $6 / 5$ constantly; gonioscopy revealed a narrow but open angle without goniosynechiae and the bulbar pressure test gave an outflow fraction of 36 per cent.

Treatment.-A peripheral iridectomy was performed on April 9, 1957, 72 hours after the congestive episode of glaucoma in the right eye had subsided. The surgical approach was made ab externo under a conjunctival flap with a von Graefe knife. The iris was grasped with forceps and a small piece abscised. Air was injected to reform the anterior chamber and eserine was instilled into the conjunctival sac.

At the first post-operative dressing the following day, the anterior chamber had recovered its normal depth and convalescence was uneventful. Scattered traces of hyphaema were recorded as being present on the anterior lens capsule in the centre of the miotic pupil and this was held to account for the immediate low post-operative visual acuity. 
Follow-up.-One month later the corrected visual acuity was still low and slit-lamp examination revealed a stippling of haemorrhage within the substance of the lens in several layers along its optical axis and not on the lens surface as was previously supposed. There was also a diffuse lens haze (Fig. 2). The upper edge of the lens was examined gonioscopically and was found to be opaque, part of the capsule being covered by a small haemorrhage. When this patch of haemorrhage had absorbed no capsular wound was visible. There has been no change in the colour or disposition of the intra-lental haemorrhage in the 7 months since the operation.

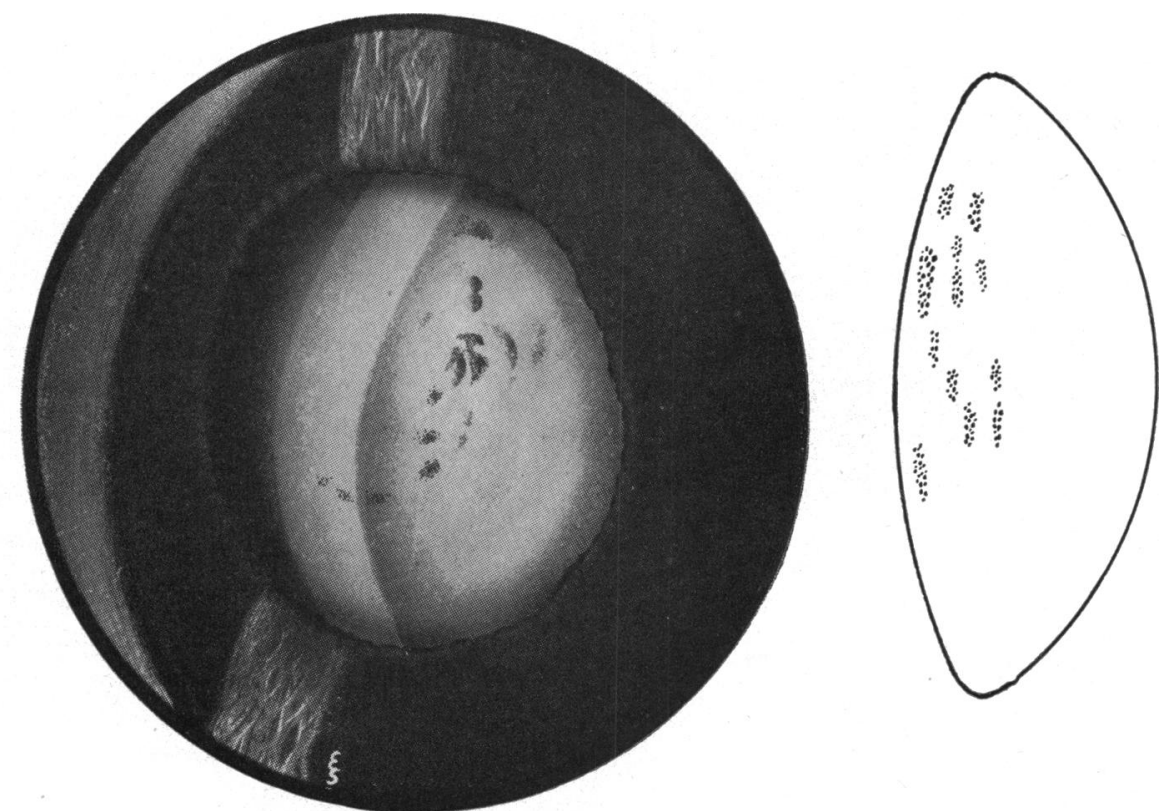

Fig. 2.-Multiple discrete haemorrhages situated at various levels in the right lens of Case 2.

\section{Discussion}

Both patients had an apparently uneventful operation for the relief of glaucoma, in which a fistula was made at the iris root. In each case haemorrhage "on the surface of the lens" was recorded soon after operation, but subsequent slit-lamp examination revealed its true position. Otherwise convalescence was without incident.

In Case 1 the fundus reflex was lost on the sixth post-operative day when a large quantity of blood suddenly appeared in the lens without any attendant hyphaema. The most likely explanation is haemorrhage from a localized area of granulation tissue which had formed around a small rupture in the upper capsule. Even so, it is remarkable that the blood could track down between the lens fibres with such apparent ease, although its passage would be facilitated if there were prior break-up of the fibres and by the formation of clefts between them. Surprising also is the absence of appreciable 
cataract change during the ensuing months. It must be assumed that the gap in the capsule has been sealed by organized fibrous tissue, the anterior face of which now fills the iridotomy.

In Case 2 there is a smaller quantity of blood within the lens. It is arranged as a number of discrete clumps situated at various depths which may indicate a different method of formation. The blood may have seeped down from the small hyphaema which was observed on the upper surface of the lens through a small capsular rupture. The gradual increasing haziness of the lens suggests that the reparative process has been less effective.

The lens is not equipped with any suitable mechanism for the removal of foreign material in bulk and very little clearing of the haemorrhage is to be expected. Stein (1955) reported a case of intra-lental haemorrhage which he attributed to bleeding from a persistent hyaloid artery; he did not observe any change in the haemorrhage during the course of one year.

\section{Summary}

Two cases are described in which blood entered the crystalline lens following the surgical treatment of glaucoma. The mode of entry is discussed. The blood has remained unchanged within the lens for 11 months in the one case and 7 months in the other.

Our thanks are due to Mr. P. L. Blaxter and Dr. A. Stanworth for permission to report these cases, and to Dr. J. L. S. Smith for criticism of the manuscript. The illustrations are by Mr. E. G. Smith of the Department of Medical Illustration, Manchester Royal Infirmary.

\section{REFERENCES}

Stallard, H. B. (1953). Brit. J. Ophthal., 37, 680.

StEIN, R. (1955). Acta med. orient. (Tel-Aviv), 14, 209. 